\title{
An Advance Paper Generator using Fuzzy Logic - A Novel Approach
}

\author{
Rashmi Dhaundiyal \\ Asst. Professor \\ Tulas Institute of Engineering \\ Dehradun, UK, India
}

\begin{abstract}
Examination is important of examining the quality of a candidate's knowledge at each phase. It test the expertise and skills of the students in any area. Hence, conducting exam at each phase may lead to some issues such as time and other resources while creating the test papers not automatically .So a computing method make the problem simpler which generates question paper automatically. Question paper generator system is implemented by considering these problems, by which paper can be generated easily. The method is based on the concept of fuzzy logic which helps in generating question paper each time with user defined complexity phase. Paper can be generated at any time. We have make use of random function so that unique paper get generated every time. Security is also maintained using MYSQL tool.
\end{abstract}

\section{Keywords}

Fuzzy Logic, Analytical, Descriptive, Fuzzification, Defuzzification

\section{INTRODUCTION}

Education is one of the important parts of our life. There are various methods by which can gain the knowledge like internet, books. Even if want to gain the knowledge through other sources like books, research articles, internet videos, some primitive knowledge is always required to get the knowledge through these resources. After gaining the knowledge it is always compulsory to examining how much capacity one has to apply in the knowledge to solve the specific problems. In the test several types of questions are mentioned .. In every of the universities question paper level is decided based on the previous years' students' result. We can have several question paper phases like easy, medium, hard etc. Professor mostly invest less time in academic work as compare to research work. Hence they generally do not want to spend much time in making the question paper .So they need automatic paper generation system which will access the questions from the database based on the contribution of all the professors of the respective subject. In this system we have flexibility for the subject experts to choose the subjects while registration. The administrator of the system can add experts details and assign subjects to them. One expert can work with maximum three subjects. They can add, remove or modify the questions. Same questions will be deleted from database system permanently if all the experts of that subject should approved for the removal of that question. We also have difficult level for question paper generation. To categorized the compelxity level of a particular question, there are two parameters, Analytics_quotient and Description_quotient. These quotients are stored as variables of the question in the database.
Analytics_quotient defines how much imagination is required to solve the question. Numerical , logical and application types of questions are generally highly analytics while theoretical questions comes under Descriptive type. If question is highly analytical, it comes under complexity level whereas descriptive questions are generally comes under easy or medium phase. Actual phase of the question is decided using both analytical and descriptive quotients. Level of the questions can be categorized after various fuzzy computations. Different people have different point of view to look at the various phases. One may feel particular question difficult while other may feel it easy. That is why fuzzy logic has been applied. Fuzzy logic works on approximation. For example, an integer we can definitely say that it can be positive or negative. But in case of complex phase of the question we can't categorize the question with an agreement by every member .So with the help of fuzzy logic we can solve this problem. The system is easy to use. At last question paper will be obtained in the doc file format which allows the experts to edit the questions. System is secure and paper can be obtained even few Minutes before of the examination which helps to avoid any paper secrecy. System works well if we have enough number of questions in the database. System can be even deployed as client server model with the database deployed on a server node.

\section{LITERATURE REVIEW}

Pratik Gadge et al[1] proposed Apriori algorithm which uses fuzzy logic. In this system login module has feature to distinguish between administrator and staff. Administrator has rights to all the information and various access rights. System supports all the possible operations like question addition, updation and deletion. System uses 'TOP E' approach which returns the topmost rows and get the limitation on number of rows. This algorithm is related to Big Data Analytics which gives priority to continous occurring of the elements. In this system paper is generated manually and get stored in text . In the Apriori algorithm, large sets of questions' are examined. So it results in large number of computations. [2] Suraj Kamya et al[2] proposed a method of the paper generated which is based on fuzzy logic. They have divided the generation into three levels. In the system all users put analytical index (a) and descriptive index (d) for the specific question. In the first level, three users put values(questions) for descriptive, easy, medium and complex parameters. Values can be anything between 1 and 20 for a and d parameters. Floating point represents the values which are also permitted. Easy and difficult values are between 1 and 5 whereas range for medium is 1 to 20 . The second level, system generates the structure based on user inputs questions. Every time new structure is 
generated. Users are allowed to put the questions into the structure generated. In the third level, question bank is created according to questions submitted by all the users . Then finally question paper is generated. System is smooth because number of users and structure can be dynamically changed. It proposes MATLAB for fuzzy logic computation. [3]Pushkar Ambole et al[4] proposed a System where question paper generated containing multiple choice questions. Fuzzy logic and ant's colony are two famous methods used in the system. In this administrator can insert the various questions into database table and their relevant options. Administrator has the rights to provide difficulty level and marks for each questions. In this administrator chooses complex level of the paper (which can be easy, medium or difficult) and generates question paper. After generation of question paper's pdf file, System makes email to the appropriate organisation. This method is mainly applied for competitive exams. [4]

\section{PROBLEM STATEMENT}

\subsection{Inputs:}

After login using ID and password, system will asked about paper pattern choices and complex level. There are three paper patterns and five difficulty levels. Persons should choose any one pattern and any one complex level.

\subsection{Output:}

Question paper in the text format will be generated.

\section{OPERATIONS OF THE SYSTEM}

\subsection{Authentication process:}

Step-1: User will registered by inserting his personal information. During registration user puts his personal information like name, sex, phone, email and any four subjects that he want to work with. Although administrator has rights to allocate different subjects. This software will generally be owned by educational institutions.

Step-2: After submitting the registration form, Message will be displayed: You will be able to login after verification by administrator.

Step-3: Administrator is person who can actually see and manage the database. We have one column in a registration table as 'Admin_verfied'.

Step-4 User or can delete his/her entry from the database. Admin can modify the personal information of the user. He can also operate the database.

Step-5: Any user's login will be completed if

he is admin ; that is if his/her entry under

the column 'Admin_verfied' is 'yes'.

\subsection{Question Accessing stage:}

Step-1: After successful login user will be permit to select those subjects for which he want to access the questions and generate the paper. At one time he can select only one subject and work with it. But he can again work with other subjects assigned to him.

Step-2 : User must choose the type of paper and difficulty level.

Step-3: After 'Fetching the questions', every questions will be appear on the user's grid view where
Analytical_quotient of the question, Descriptive_quotient , Marks, paper generation. (' 1 ' for included, ' 0 ' for not included) can be checked.

\subsection{Paper Generation:}

Step-1: After clicking on 'Generate paper' paper should be created and stored into pdf/doc file.

Step-2: User should also change the questions.

Step-3: With the use of 'Random function', the question paper generated will be different every time.

\subsection{Question insertion:}

Step-1: Like the paper final creation, a different interface will be provided for question insertion.

Step-2: User should enter the Question, its Analytical_quotient, Descriptive_quotient and Marks.

Step-3: Before insertion user can see all the questions in the database, so that redundancy should be avoided. Different button is provided to check all the questions.

\subsection{Question parameters modification}

Step-1: User can update the Analytical_quotient, Descriptive_quotient of the questions.Previously quotients and manual entered quotients will be stored into database

Step-2: The reason for allocating average of these parameters is we want to take every user's opinion into consideration.

\subsection{Question Removal}

Step-1: User can also remove the outdated questions. However after pressing the remove button for each question, it is not removed immediately. It will wait until the coming of all users' reply for that question.

Step-2: One column named as Remove in the table of every subject's table database. When specifc user removes the question then deletion count for that question in the subject's table get increment its value by one. Also the entry in the $\log$ is made automatically so that this user should not be able to increment the removal count for a particular question not more than one time.

Step-3: After verifying with all the users for the particular question for deletion, question will be removed from the database .

\section{PROPOSED ALGORITHM}

1. Start

2. Analyze the subject and maximum marks.

3. Follow the steps 4 to 19 for each question.

4. For each in a database, read analytical_quotient and Descriptive_quotient.

5. Allocate these values into variables $m$ and $n$ such that $\mathrm{m}=$ analytical_quotient, $\mathrm{n}=$ Descrptive_quotient.

6. Find the range of ' $\mathrm{m}$ ' from fuzzy graph or fuzzy equations. These are the fuzzification equations .

7. Compute ' $v$ ' values (' $v$ ' is membership value of input variable to the particular set) for $\mathrm{m}$. Each range has one or two membership equations.

8. Follow the steps 6 and 7 for ' $n$ ' values .

9. We can get one or two of ' $v$ ' values for m.Same for the 
'n'.

10. We can form total one, two or four groups depending upon number of ' $v$ ' values that we got for ' $m$ ' and ' $n$ ' in the previous steps. Every group contains two ' $v$ ' values, one for ' $\mathrm{m}$ ' and one for ' $\mathrm{n}$ '. We consider all possible groups (example: If we get 2 ' $\mathrm{v}$ ' values for ' $\mathrm{m}$ ' and 2 for ' $n$ ' then groups formed are (vm1, vn1), (vm1, vn2), (vm2, vn1), (um2, un2))

11. After all the groups creation, take minimum value from each group. Thus every group now contains only one ' $\mathrm{v}$ ' value either 'vm' or 'vn'.

12. Take maximum value from the values we got in the step 11.

13. The specific value can accessed in the last step corresponds to a relevant group among all the groups formed.

14. Take vm and vn values of that group. These ' $v$ ' values corresponds to a particular rule in fuzzy rule base.(Example: IF high analytical_quotient and medium Descriptive_quotient THEN complex)

15. Using proper fuzzy rule we get question level or range(each range in fuzzy output equations)

16. For input equations, we also have fuzzy output membership equation. By checking the exact range(depending on value got in step 15) and applying corresponding output equation, we get question level in the defuzzified form.

17. Follow step 16 for each range if any.

18. Take average of all defuzzified integer values. This value will decide the level of the question.

19. If level selected by the user equals to the level got in previous step question will be marked as ' 1 '(can include) otherwise ' 0 '(cannot include).

20. Stop after visiting all the questions.

\section{FUZZY EQUATIONS}

\section{1 :Membership method for Analytical approach}

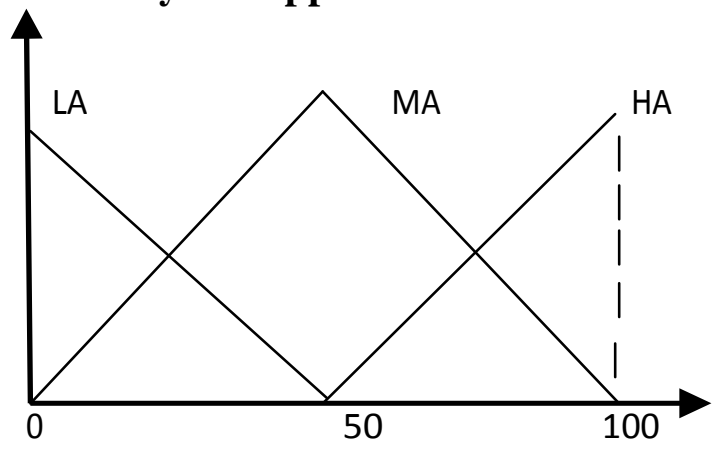

$$
\begin{aligned}
& \mathrm{uLA}=(50-\mathrm{x}) / 50 \\
& \begin{aligned}
\mathrm{x}< & =50 \mathrm{uMA}=\mathrm{x} / 55 \\
=\mathrm{x}< & =50 \\
= & (100-\mathrm{x}) / 45
\end{aligned}
\end{aligned}
$$

$$
\begin{aligned}
& =x<=100 \text { uHA }=(x-50) / 45 \\
& x<=100 \\
& 50<=
\end{aligned}
$$

6.2 : Membership method for 'Descriptive' approach

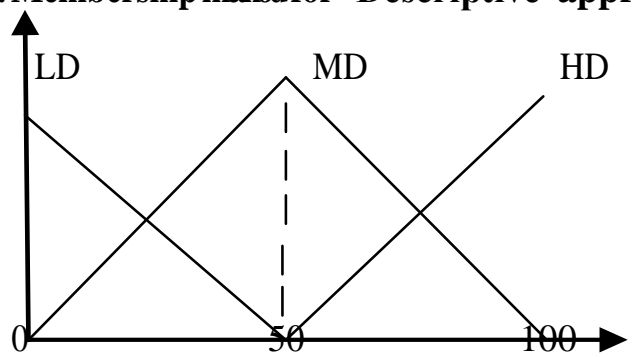

$\mathrm{uLD}=(50-\mathrm{y}) / 500<=\mathrm{y}<=50$

$\mathrm{uMD}=\mathrm{y} / 500<=\mathrm{y}<=50$

$=(100-\mathrm{y}) / 5050<=\mathrm{y}<=100 \mathrm{uHD}=(\mathrm{y}-50) / 4550<=\mathrm{y}<=100$

\section{3 : Output}

\section{Function ( to decide question level)}

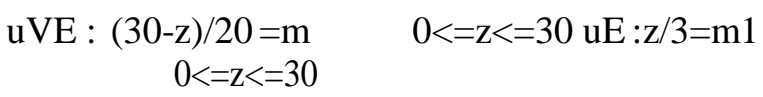

$(40-) / 30=\mathrm{m} 2 \quad 30<=\mathrm{z}<=40$

$\mathrm{uM}: \quad(\mathrm{z}-30) / 30=\mathrm{m} 1 \quad 30<=\mathrm{z}<=40 \quad(50-\mathrm{z}) / 30$

$=\mathrm{m} 240<=\mathrm{z}<=50$

$$
\begin{aligned}
\mathrm{uH}: & (\mathrm{z}-40) / 30 \\
= & \mathrm{m} 1 \\
40<\mathrm{z}< & =30(100- \\
\mathrm{z}) & / 30 \\
= & \mathrm{m} 250<=\mathrm{z}<= \\
& 100
\end{aligned}
$$

uVH: $(\mathrm{z}-50) / 40=\mathrm{m} \quad 50<=\mathrm{z}<=100$

Final paper is displayed in the text file format which includes subject name and maximum marks.

\section{IMPLEMENTATION}

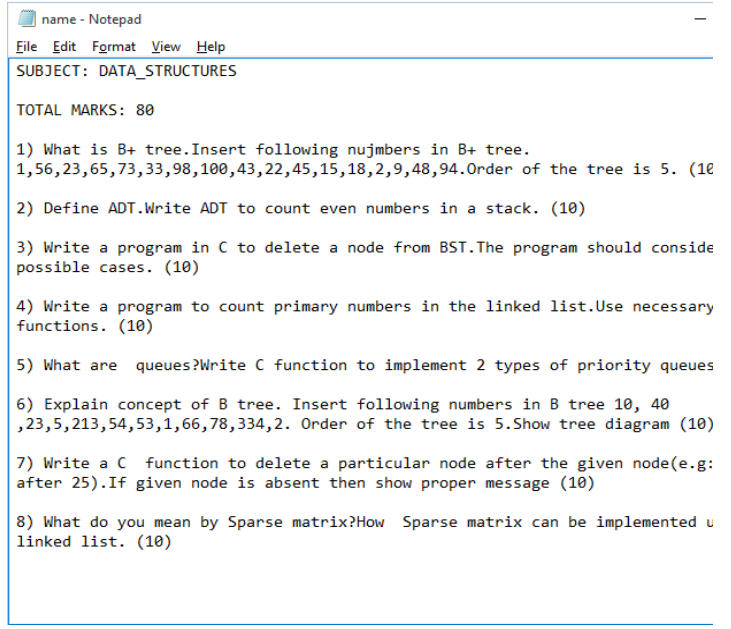




\section{CONCLUSION}

With this paper we present an 'Advanced Question paper Generator' implemented using fuzzy logic. The System is easy to use and it generates the question paper in very few seconds.

Paper generated is in the text format, due to this user can make alteration. This system is dynamic and it supports all necessary operations. Functionalities such as login and registration make the system much secure. This System is able to generate paper on the examination day within a few seconds so that any paper secrecy can be maintained.

\section{REFERENCES}

[1] Pratik Gadge, Ravikant Vishwakarma, Divya Gandhi, "Advanced Question Paper Generator Using Fuzzy Logic", International Research Journal of Engineering and Technology (IRJET), 2017, volume-4 issue-3, page 455 .

[2] Suraj Kamya, Madhuri Sachdeva, Navdeep Dhaliwal and Sonit Singh, "Automated Question Paper Generator System using Apriori Algorithm and Fuzzy
logic",International Journal for Innovative Research in Science \& Technology, (IJIRST), 2016.

[3] S. Kamya, M. Sachdeva, N. Dhaliwal, and S. Singh, "Fuzzy logic based intelligent question paper generator", IEEE Advance Computing Conference (IACC), 2014.

[4] Pushkar Ambole, Urvashi Sharma, Pushkar Deole "Intelligent Question Paper Generation System", International Journal of Scientific and Technical Advancements(IJSTA)

[5] S.N.Sivanandam, S.N.Deepa "Principles of Soft Computing" Second Edition, WileyPublication

[6] Bard, J. F., Balachandra, R., and Kaufmann, P.E. (1988)An interactive approach to $R \& D$ project selection and termination,, IEEE Transactions on Engineering Management 35, pp.139-146.

[7] Cooper, R. G., Edgett, S. J., and Kleinschmidt, E. J. (2002) „Optimizing the stage-gate process: What bestpractice companies do-II,, Research Technology Management 45(6), pp. 43. 